Revue d'histoire de l'Amérique française

\#HE REVUE D.HISTOIRE DE L'AMÉRIQUE FRANÇAISE

\title{
Le gouvernement des Trois-Rivières sous le régime militaire (1760-1764) \\ Inventaire du gouvernement
}

\section{Marcel Trudel}

Volume 5, numéro 1, juin 1951

URI : https://id.erudit.org/iderudit/801685ar

DOI : https://doi.org/10.7202/801685ar

Aller au sommaire du numéro

Éditeur(s)

Institut d'histoire de l'Amérique française

ISSN

0035-2357 (imprimé)

1492-1383 (numérique)

Découvrir la revue

Citer cet article

Trudel, M. (1951). Le gouvernement des Trois-Rivières sous le régime militaire (1760-1764) : inventaire du gouvernement. Revue d'histoire de l'Amérique française, 5(1), 69-98. https://doi.org/10.7202/801685ar d'utilisation que vous pouvez consulter en ligne.

https://apropos.erudit.org/fr/usagers/politique-dutilisation/ 


\title{
LE GOUVERNEMENT DES TROIS-RIVIERES SOUS LE REGIME MILITAIRE*
}

\section{0-1764}

\author{
IN VENTAIRE DU GOUVERNEMENT
}

L'arrivée de puissants renforts anglais à Québec permettait désormais à Murray de porter la guerre en amont et d'essayer d'opérer sa jonction avec les armées des lacs Champlain et Ontario. Le 11 juillet 1760 , il partit donc de Québec avec une flotte de trente-cinq voiles, portant "trois mille hommes de troupes, non compris les matelots, une prodigieuse quantité de vivres, de munitions et toute l'artillerie nécessaire pour une expédition considérable"'. A cette flotte qui menaçait sans cesse de dépasser les Français qui retraitaient par terre, on tenta en vain de bloquer le passage a Deschambault et à Grondines: la flotte remontait toujours.

Pourrait-on, au moins, l'arrêter dans les limites du Gouvernement des Trois-Rivières? "Si nos navires, avait écrit La Pause en novembre 1759 , ne peuvent arrêter ceux de l'ennemi à la batture de Batiscan, il faut du moins qu'ils n'abandonnent cette position qu'a la dernière extrémité; l'armée doit se replier dans le même temps, attendu qu'elle ne pourrait s'y soutenir si l'ennemi était maître de la rivière""2: à la fin de juillet, la flotte était rendue à Batiscan et à Champlain. Le 6 aout, les Anglais débarquent à Champlain au nombre de 86, "mais, rapporte Vaudreuil, ils rembarquèrent sitôt qu'ils aperçurent quelques miliciens à cheval qui les allaient reconnấ-

* Extrait d'un ouvrage en préparation: Le régime militaire, 1759-1764.

1. Collection des Manuscrits du Maréchal de Lévis (publiée sous la direction de l'abbé H.R. Casgrain) (15 vol., Montréal, 1889-1895); XI: 249. Collection citée à l'avenir sous le titre Coll. Lévis.

2. Rapport de l'Archiviste de la province de Québec [RAPQ] pour 1993-1994 (Québec, 1934), 138. 
tre"'3; le même jour, ils sont à Bécancour, sur la rive sud, et Knox inscrit dans son journal, à cette date: "Les habitants de la paroisse de Bécancour sont devenus sujets de Sa Majesté Britannique". D'ailleurs, “cette flotte, à mesure qu'elle avançait, envoyait désarmer les habitants des paroisses, qu'ils laissaient derrière, donnant des manifestes pour faire poser les armes aux habitants, menaçant d'incendier les maisons de tous ceux qui n'y rentreraient pas et qui n'y seraient pas trouvés, ou qui seraient pris les armes à la main"s. Plusieurs navires s'attardèrent dans les environs de Champlain, sans doute pour assurer la reddition des paroisses, cependant que le reste de la flotte remontait toujours le fleuve.

On prit des mesures pour défendre la ville des Trois-Rivières: "le sieur Dumas, qui côtoyait la flotte anglaise, se pressa d'y arriver pour y construire quelques batteries et retranchements, qui furent dirigés par le sieur de Pontleroy, ingénieur en chef, et le sieur de Montbeillard, commandant l'artillerie. Il avait ordre de défendre cette ville, si l'ennemi entreprenait de l'attaquer"' Mais Murray négligea complètement les Trois-Rivières ou, pour reprendre le mot de Knox, "la capitale insignifiante de ce Gouvernement tant vante" " le 8, une partie de la flotte passa devant la ville pour aller mouiller à quelques milles en amont ${ }^{8}$. Trois jours plus tard, la ville est encore sous la domination française, mais ce ne peut plus être pour longtemps, ainsi que l'écrit le frère du grand-vicaire Perrault: "L'on a retranché la ville où je suis, mais on a rappelé les troupes. Il ne reste que quatrevingts soldats et une cinquantaine de Canadiens. La seconde division de vingt-deux bâtiments qui est au Cap, a, dit-on, 1,200 hommes. Ils ont fait rendre les armes aux habitants de Batiscan, à la réquisition du capitaine... Les dits habitants se trouvant maîtres ont été trouver le commandant anglais avec prière de les recevoir sous sa domina192.

3. Vaudreuil au ministre, 11 aout 1760, Archives des Colonies [ AC] F3 16:

4. John Knox, An Historical Journal of the Campaigns in North America, for the Years 1757, 1758, 1759 and 1760, edited with introduction, appendix and index, by Arthur G. Doughty (3 vol., Toronto, The Champlain Society, 1914), II: 484.

5. RAPQ (1931-1932), 120 (Relation de Poularies).

6. "Relation anonyme", Coll. Lévis, XI: 250.

7. Knox, Journal, II: 487.

8. RAPQ (1931-1932), 120 (Relation de Poularies). 
tion. Les habitants du Cap en ont fait autant; les habitants de Bécancour ont été a bord dire que les troupes avaient évacué les Trois-Rivières et qu'il n'y reste personne. Ainsi, vous voyez que nous serons bientôt anglais."

La Pause avait prévu, dès 1759 , que l'armée ne serait point en sûreté aux Trois-Rivières ni à la Pointe-du-Lac, qu'on pourrait cependant se retrancher successivement derrière les rivières Yamachiche, du Loup, Maskinongé et Berthier, mais, avait-il ajouté, "le passage de toutes ces rivières ne peut être disputé longtemps, étant fort étroites"10. Il y avait une dernière ressource: "boucher les chenaux de la sortie du lac Saint-Pierre"11; l'étroitesse de ces chenaux et le grand grand nombres d'îles serrées qui, nous dit Franquet, couvrent quatre lieues ${ }^{12}$, semblaient favoriser cette opération d'une façon très avantageuse. Bourlamaque se mit en frais de l'exécuter; Lévis alla même se rendre compte de l'état des travaux ${ }^{13}$, mais Bourlamaque, "voyant qu'il ne pouvait avoir fini à temps de fermer les chenaux et craignant de ne pouvoir retirer les troupes qui étaient dans les îles, si les ennemis passaient, les replia à Sorel"; le 12 août, la première division de la flotte anglaise passait les chenaux ${ }^{14}$. La dernière tentative sérieuse pour empêcher l'avance des Anglais, avait échoué: il ne restait plus pour les Canadiens qu'à attendre le sort des opérations de Montréal. Le 8 septembre suivant, Vaudreuil était contraint de capituler, et tout le Canada passait sous le régime militaire, en attendant que les métropoles décident définitivement de la possession de la colonie.

Le régime militaire ne changea rien aux grandes divisions"administratives adoptées par les Français, le pays resta partagé en trois Gouvernements: Québec, Trois-Rivières et Montréal.

Le moins considérable était celui des Trois-Rivières. Comme les Gouvernements voisins, il se trouvait coupé en deux parties par le

9. Voir Chanoine Prosper Cloutier, Histoire de la paroisse de Champlain (2 vol., Trois-Rivières, 1915), II: 166s, lettre de Louis Perrault, 11 aout 1760.

10. RAPQ (1933-1934), 138.

11. RAPQ (1931-1932), 119.

12. Franquet, Voyages et Mémoires sur le Canada (Québec, 1889), 25.

13. Lévis à Belle-Isle, 7 aout 1760, Coll. Lévis, II: 376.

14. RAPQ (1931-1932), 120s. 
fleuve, mais alors que les Gouvernements de Québec et de Montréal avaient d'une rive à l'autre plusieurs points de contact assez rapprochés, le Gouvernement des Trois-Rivières n'en avait aucun: le fleuve s'y trouvait très large sur tout son parcours et le lac Saint-Pierre en éloignait encore davantage les rives l'une de l'autre; la longue et dangereuse traversée du fleuve, faite la plupart du temps sur de petits canots, compliquait sérieusement les relations des gouvernants avec leurs administrés. Par contre, le Gouvernement des Trois-Rivières était beaucoup plus étroit que les deux autres: sur la rive nord, depuis Sainte-Anne-de-la-Pérade jusqu'à Maskinongé qui en marquaient les limites extrêmes, il mesurait quelque soixante milles; sur la rive sud, d'Yamaska à Saint-Pierre-les-Becquets, il en mesurait à peu près autant. Quant à sa profondeur, elle n'était pas mieux définie que celle de ses voisins: elle s'étendait vaguement vers les terres de la Compagnie de la Baie d'Hudson et, suivant la description plutôt étrange de Burton, "jusqu'à la Nouvelle-Écosse, le New-Hampshire et le Massachusetts" 15 . Voyons quel est l'état de ce Gouvernement, lors de son occupation par l'armée anglaise à l'automne de 1760 .

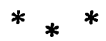

La première seigneurie que l'on rencontre sur la rive nord, en pénétrant dans le Gouvernement des Trois-Rivières par l'est, c'est la seigneurie de Sainte-Anne-Est, ou Dorvilliers parce qu'elle appartient à François Chorel d'Orvilliers: elle a un front de trois quarts de lieue et sa profondeur ne dépasse pas une lieue ${ }^{16}$. Jusqu'en 1695, elle avait constitué une seule et même seigneurie avec sa voisine, Sainte-Anne-Ouest: cette dernière qui, dans les débuts, avait le même front et la même profondeur, s'était vu agrandir de trois aug-

15. Documents relatifs d̀ l'Histoire constitutionnelle du Canada 1759-1791 Choisis et édités avec notes par Adam Shortt et Arthur G. Doughty. (Archives publiques, Ottawa, 1921), 67. (Désormais abrégé comme suit: Doc. const.)

16. P.-G. Roy, Inventaire des concessions en fief et seigneurie, fois et hommages et aveux et denombrements, conservés aux Archives de la Province de Québec (6 vol., Beauceville, 1927-1929), II: 145-154; Joseph Bouchette, Description topographique de la Province du Bas-Canada, avec des remarques sur le Haut-Canada, et sur les relations des deux provinces avec les Etats-Unis d'Amérique (Londres, 1815), 323s.; Autrefois et aujourd'hui à Sainte-Anne de la Pérade - Jubilé sacerdotal de Mgr des Trois-Rivières (Trois-Rivières, 1895), 38-40; Chanoine Prosper Cloutier, Histoire de la paroisse de Champlain ( 2 vol. Trois-Rivières, 1915), II: $418 \mathrm{~s}$. 
mentations successives, de sorte qu'en 1760 sa profondeur se rend jusqu'à huit lieues et demie du fleuve, en couvrant de sa largeur tout le terrain compris entre les seigneuries de Grondines et de Batiscan ${ }^{17}$. Elle appartient à Charles-François-Xavier Tarieu de Lanaudière, passé en France avec les troupes de Lévis ${ }^{18}$. Sur la rive occidentale de la rivière Sainte-Anne, commence une seigneurie beaucoup plus petite, celle de Sainte-Marie, trois quarts de lieue de front par une demi-lieue de profondeur, soit depuis l'île des Pins inclusivement jusqu'à la frontière de la seigneurie de Batiscan: Louis-Joseph Gastineau en est le seigneur en titre ${ }^{19}$. Le sol de ces seigneuries, écrira Bouchette, "est passablement fertile et consiste en une terre légère et sablonneuse sur un argile rougeâtre vers le front; mais en avançant vers le fond, on trouve un mélange de marne jaune et de terre noire"; les augmentations "sont presque toutes en forêts qui produisent du bois de construction de toute espèce, dont une partie est d'une excellente venue et d'une grande valeur" ${ }^{20}$. Sur 8,487 acres concédées, on en fait valoir 1,950 et l'on cultive surtout du blé et de l'avoine; le bétail comprend 130 chevaux, 252 bêtes à cornes et 208 moutons ${ }^{21}$. Ces trois seigneuries composent la paroisse de Sainte-Anne ${ }^{22}$, dont François Rouillard est le curé ${ }^{23}$ : il vit dans un presbytère de pierre qui date de 1739 ; l'église de 1716 vient de subir des réparations

17. Roy, ibid., Bouchette, ibid.

18. Pierre-Georges Roy, La famille Tarieu de Lanaudière (Lévis, 1922), 74. Il reviendra au Canada en 1763.

19. P.-G. Roy, Inventaire des concessions en fief et seigneurie... III: 11-14.

20. Joseph Bouchette, Description topographique de la Province du Bas-Canada, 324. Cette description est de 1815, mais nous n'en extrayons que les données qui peuvent correspondre à celles de 1760 .

21. Ce tableau des terres en valeur et du bétail (Doc. const., 76) est un rapport signé en 1762, mais tout nous laisse croire qu'il a été rédigé en même temps que le recensement de 1760; le recensement de 1762 n'est que celui de 1760 auquel on a ajouté les changements survenus dans l'intervalle.

22. A moins d'indications contraires, nous référons, une fois pour toutes, aux Edits, Ordonnances royaux, declarations et Arrêts du Conseil d'Etat du Roi concernant le Canada, vol. I (Québec, 1854): 443-462, pour les limites des paroisses.

23. Il meurt peu de temps après le recensement, soit en décembre 1760 , remplacé par Louis-Michel Guay qui restera curé de cette paroisse jusqu'en 1785, date de sa mort. Voir R.P. Alexandre Dugré, s.j., La Pointe-du-Lac "Pages trifluviennes", Série A, no 15 ('Trois-Rivières, 1934), 39; Autrefois et aujourd'hui à Sainte-Anne de la Perade - Jubilé sacerdotal de Mgr des Trois-Rivières ('Trois-Rivières, 1895), 43-45. 
considérables: on y remarque un retable d'autel du sculpteur Bolvin et la dorure est de Le Vasseur ${ }^{24}$. Le recensement de 1760 donne 528 habitants, logés dans 101 maisons $^{25}$. Le chemin du roi est coupé par la rivière Sainte-Anne qu'il faut franchir en canot ou en bac: "la rivière est si basse que les grands bateaux se conduisent avec des perches" ${ }^{26}$.

Immédiatement à l'ouest, la seigneurie de Batiscan, concédée aux Jésuites dès 1639, s'étend sur un front de deux lieues, depuis la seigneurie de Sainte-Marie jusqu'à la rivière Champlain; elle pénètre jusqu'à vingt lieues à l'intérieur des terres ${ }^{27}$ : aucune autre seigneurie de la Nouvelle-France ne dépasse cette profondeur. Dans les parties basses, le sol "offre une terre légère, un peu sablonneuse, sur une couche de bonne argile; mais, en avançant au nord, il devient plus fort, et pendant un espace considérable, il est enrichi par une belle terre noire... On n'a pas encore examiné cette seigneurie dans toute sa profondeur, mais aussi loin qu'on l'a visitée, on a trouvé qu'elle produisait d'excellent bois de construction de différentes espèces" ${ }^{\prime 28}$. Elle est arrosée par la rivière Batiscan et, dans sa profondeur, traversée par la rivière Saint-Maurice. On avait découvert du minerai de fer en cet endroit dès 1660 et, en 1735, on avait songé sérieusement à l'exploiter ${ }^{29}$ : en 1760 , il n'y a encore rien de fait. La seigneurie de Batiscan compte deux paroisses, Batiscan et Sainte-Geneviève. Batiscan est située à l'embouchure de la rivière du même nom; la paroisse, dont Pierre Maugue-Garaut de Saint-Onge est le curé, a son église de pierre; Joseph Rouillard y exerce les fonctions de notaire; elle compte 189 habitants et 38 maisons; les paroissiens

24. Autrefois et aujourd'hui à Sainte-Anne de la Pérade... 41-43.

25. Le recensement de septembre 1760 est reproduit dans RAPQ (1946-1947), 5-53 et dans Ravport des Archives du Canada [ RAC] (1918), app. B, 158-189. Il mentionne comme paroisses distinctes, Ste-Anne et Ste-Marie (RAPQ (1946-1947), 29-33), mais elles ne forment, en réalité, qu'une seule paroisse. 326.

26. Joseph Bouchette, Description topographique de la Province du Bas-Canada,

27. P.-G. Roy, Inventaire des concessions en fief et seigneurie... I: 170-173; Bouchette, ibid., 320-323.

28. Bouchette, ibid., 321.

29. Benjamin Sulte, Les Forges Saint-Maurice, "Mélanges historiques" no 6, (1920), 16 et 49. 
ont mis en valeur 1,482 acres sur les 4,033 concédées, ils possèdent 46 chevaux, 127 bêtes à cornes et 38 moutons $^{30}$. La rivière Batiscan, comme la rivière Sainte-Anne, doit être franchie en canot ou en bac $\mathrm{c}^{31}$. Quant à Sainte-Geneviève (alors appelée Rivière-Batiscan), elle est établie en amont de la rivière; une partie de ses habitants ont déjà atteint la rivière des Envies ${ }^{32}$. Le recensement donne 515 habitants qui ont mis en valeur le septième des 3,500 acres qu'on leur a concédées; ils ont une centaine de maisons, 107 chevaux, 240 bêtes à cornes et 25 moutons; dans l'une et l'autre de ces paroisses, on cultive surtout du blé et $d u$ tabac $^{33}$. L'église et le presbytère sont tout neufs: ils datent d'une couple d'années ${ }^{34}$. Antoine Lagroix dessert la paroisse depuis $1749^{35}$.

$\mathrm{Au}$ delà de la petite rivière Champlain, nous entrons dans la seigneurie de Champlain (une lieue et demie par quatre) dont le seigneur en titre est Joseph-Antoine Pezard de Champlain ${ }^{36}$. Sur les 5,660 acres concédées, 1,400 sont mises en valeur; le sol se prêterait admirablement bien à la culture du lin, mais on ne cultive surtout que le blé et le tabac ${ }^{37}$. La paroisse de Champlain, qui couvre la seigneurie, a 216 habitants, répartis dans 50 maisons; le bétail se chiffre à 80 chevaux, 175 bêtes à cornes et 10 moutons $^{38}$. L'église

30. RAPQ (1946-1947), 25, 52-53; Doc. const., 76; Chan. P.A.A. Bellemare et Hervé Trudel, ptre, Batiscan (Trois-Rivières, 1933), 21s.; Joseph-Edmond Roy, Histoire du notariat au Canada depuis la fondation de la colonie jusqu'd nos jours (4 vol., Lévis, 1899-1902), II: 11. Le curé Saint-Onge, né à Montréal en 1721, deviendra chanoine, puis vicaire général; mort aux Trois-Rivières en 1795.

31. Bouchette, op. cit., 322.

32. Se déverse dans le Batiscan à l'endroit connu sous le nom de Saint-Stanislas; les habitants de la Rivière-des-Envies avaient même demandé une église en 1756 (Abbé Auguste Gosselin, L'Eglise du Canada depuis Mgr de Laval jusqu'd la conquête (3 vol., Québec, 1911-1914) III: 433).

33. RAPQ (1946-1947), 26-28, 52s; Doc. const., 76.

34. E.-Z. Massicotte, Sainte-Geneviève-de-Batiscan (Trois-Rivières, 1936), 188.

35. Remplacé par Sainte-Onge en 1761 (Massicotte, ibid., 56).

36. P.-G. Roy, Inventaire des concessions en fief et seigneurie... II: 105-109; Joseph Bouchette, Description topographique... 319-320; Chan. Prosper Cloutier, Histoire de la paroisse de Champlain, f: $340 \mathrm{~s}$.

37. Doc. const., 76.

38. RAPQ (1946-1947), 22s, 52s. 
est en pierre et c'est François Morisseau-Boismorel qui y exerce les fonctions curiales; Champlain a l'avantage très rare de posséder un pensionnat tenu par deux Sœurs de la Congrégation; le chirurgien Egon et le notaire Nicolas Duclos pratiquent dans la paroisse ${ }^{39}$. Entre cette seigneurie et la rivière Saint-Maurice, les Jésuites possèdent une autre seigneurie, celle du Cap-de-la-Madeleine, qui mesure deux lieues par vingt; cette seigneurie et celle de Batiscan dépassent de loin en profondeur toutes les seigneuries que l'on connait $^{40}$. On y avait découvert du minerai de fer en 1650; un expert avait déclaré qu'on ne pouvait "désirer meilleur fer et en plus grande abondance" et, en 1743, on avait inclus les mines du Cap dans le domaine que la Compagnie des Forges devait exploiter ${ }^{41}$. De plus, Sarrazin avait, en 1732, longuement examiné onze sources d'eaux minérales dont il faisait les plus grands éloges ${ }^{42}$. La paroisse, qui porte le nom de la seigneurie, compte seulement 160 habitants et 33 maisons; on exploite 600 acres sur les 2,100 qui ont été concédées; les habitants possèdent 41 chevaux, 142 bêtes à cornes et 19 moutons; la culture est en avoine et en $\operatorname{tabac}^{43}$. La petite église de pierre n'a pas de curé résidant: elle est desservie par le curé de Champlain, Morisseau-Boismorel ${ }^{44}$.

Pour franchir le Saint-Maurice, il faut encore utiliser le canot ou le bac "en dessus des îles situées à son débouché dans le fleuve"; l'hiver, il faut remonter la rive gauche, "au moins une demi-lieue", descendre ensuite sur la rivière qu'on remonte sur la glace avant de la franchir "à l'endroit nommé Toutrecaut", puis remonter l'escarpement pour aller rejoindre le chemin $d u$ roi $^{45}$. En traversant la

39. Abbé Eddie Hamelin, La paroisse de Champlain (Trois-Rivières, 1933), 70ss; Chan. Prosper Cloutier, Histoire de la paroisse de Champlain, I: 213s, 455ss; J.-Edmond Roy, Histoire du notariat... II: 11.

40. P.-G. Roy, Inventaire des concessions en fief et seigneurie... II: 1-10; Bouchette op. cit., 316-319.

41. Sulte, Les Forges Saint-Maurice, 16, 18, 89.

42. Ibid., 47.

43. RAPQ (1946-1947), 20s, 52s; Doc. const., 76.

44. P.-E. Breton, O.M.I., Cap de la Madeleine, ciie mystique de Marie, (TroisRivières, 1937), 99 et 109. L'église de 1760 est le petit sanctuaire d'aujourd'hui, moins la sacristie construire en 1762 .

45. Franquet, Voyages et Mémoires sur le Canada (Québec, 1889), 134-136. 
rivière, nous remarquons les îles de l'embouchure qui ont été concédées en fiefs dès le dix-septième siècle: l'île Saint-Quentin; l'île de la Poterie, fief noble de cent arpents; l'île Saint-Christophe, fief de quatre-vingts arpents donné en franche-aumône aux Jésuites; l'île Saint-Joseph, fief noble de soixante arpents; les terres de ces iles sont mises en valeur depuis longtemps ${ }^{46}$.

Sur la terre ferme, dans la ville des Trois-Rivières et dans les environs, presque tout le terrain est concédé en fiefs: le fief Hertel est le premier que nous trouvons en passant de l'embouchure du SaintMaurice vers la ville, fief noble de cinquante arpents dont la concession date de 1633; c'est sur une partie de ce fief que se trouve construit le couvent des Ursulines ${ }^{47}$. Au nord du fief Hertel, le Haut-Boc, dont la concession, aussi de 1633, comprend à peu près la même étendue ${ }^{48}$. Sur le Platon, le fief Pachirini, quatre arpents concédés d'abord à l'Algonquin Charles Pachirini, passés plus tard aux Jésuites ${ }^{49}$; le fief La Poterie, au nord de l'église paroissiale, propriété de la famille Boucher de Niverville qui possède, rue Bonaventure, un manoir tout récent $t^{50}$; le Coteau Saint-Louis qui couvre 98 arpents, fief des Jésuites ${ }^{51}$; à l'ouest de ce fief, la seigneurie SainteMarguerite, avec son front de trois quarts de lieue sur une lieue de profondeur, propriété de la famille Chastelain ${ }^{52}$, et, enfin, en revenant vers le fleuve, la seigneurie de la Commune, concédée aux Habi-

46. "Les îles de l'embouchure du Saint-Maurice", par Montarville Boucher de la Bruère, dans Chapelles et églises trifluviennes. (Les Trois-Rivières, 1933), 31-45.

47. P.-G. Roy, Inventaire des concessions... I: 27-31. Champlain avait d'abord concédé 200 arpents, puis n'en donna que cinquante.

48. Benjamin Sulte, Trois-Rivières d'Autrefois, "Mélanges historiques", no 18 (Montréal, 1931), 71. Les 200 arpents de la concession de 1633 furent réduits à peu près comme ceux de Hertel; selon le cadastre de 1863, on n'aurait plus que 35 arpents (Cadastres abrégés des seigneuries des Trois-Rivières, no 39).

49. P.-G. Roy, Inventaire des concessions... I: 282-284; Revue canadienne (1908), 491 et 564. Situé à peu près entre les rues St-Louis, St-Antoine et Notre-Dame.

50. Ibid., II: 71 et 75. Entre les rues Bonaventure, Laviolette, St-Pierre et StJoseph. Le manoir est de 1756.

51. Ibid., I: 45-48. Borné par Notre-Dame, des Forges et, au nord-est, par le fief La Poterie; son front, sur la rue Notre-Dame n'était que de 450 pieds, mais le fief allait ensuite en s'élargissant; dans les profondeurs, il avait une largeur de trois arpents.

52. Ibid., III: 185-189; Bouchette, Description topographique..., 305-311. Bornée par la rivière St-Maurice au nord-est, par Vieux-pont au sud-ouest, sa pr l s'étend jusqu'aux seigneuries de la Pointe-du-Lac et de Saint-Maurice. 
tants des Trois-Rivières "pour servir de pâturage à leur bestial",53. Le centre paroissial de ces fiefs est Trois-Rivières, capitale du Gouvernement.

De capitale, elle n'en a pour ainsi dire que le nom, puisqu'un bon nombre de paroisses rurales des Gouvernements de Québec et de Montréal la surpassent en population et qu'elle-même ne dépasse que très difficilement certaines paroisses de son Gouvernement, comme Yamachiche, Sainte-Anne et Sainte-Geneviève. La ville n'a que 586 habitants et 110 maisons $^{54}$. Son importance vient de ce qu'elle "est en quelque façon l'entrepôt de la communication" de Québec à Montréal $1^{55}$, ce qui fera dire à Haldimand: "Ma Maison étoit comme la seule Taverne qu'il y eât pour toute l'armée entre Québec \& Mont Real" ${ }^{56}$. Elle est située, écrit Kalm, au sommet d'un plateau sablonneux, et occupe un site des plus agréables, entre la rivière qui coule à ses pieds et dont le lit a en cet endroit une largeur d'un mille et demi, et de beaux champs de blé, qui paraissent très fertiles, quoique le sol contienne beaucoup de sable"57. Cette "petite ville de marche" ou ce "grand village" 58 'a rien d'une place fortifiée: "Présentement cette place est dépourvue de moyens de défense; les Français comptant sur une protection imaginaire ont complètement négligé de fortifier la ville et laissé les habitants construire leurs maisons près de la commune, sur un terrain bas exposé aux inondations chaque printemps, au lieu de tirer parti de l'endroit élevé sur lequel se trouve située une partie de la ville"59. Jusqu'en 1752, elle avait été "fermée d'une enceinte de pieux de 10 à 12 pouces de diamètre sur 12 pieds de hauteur" ${ }^{60}$, mais un incendie l'avait détruite. Nous entrons dans

53. Ibid., I: $285 \mathrm{~s}$.

54. RAPQ (1946-1947), 5-9, 52s.

55. Franquet, Voyages et Mémoires sur le Canada, 109.

56. AC, B 21-1: 192.

57. Voyage de Kalm en Amérique, "Mémoires de la Société historique de Montréal", huitième livraison, 65.

58. Ibid.

59. Doc. const., 69, Rapport de Burton.

60. Franquet, Voyages et Mémoires sur le Canada, 110. D'après Sulte, cette enceinte serait aujourd'hui un carré formé par les rues St-Pierre, St-Jean, St-Louis, des Casernes et le coude de Notre-Dame (Trois-Rivières d"autrefois, "Mélanges historiques, no 18,25 et 36 .) 
une ville ouverte, aux rues "tortueuses" et où, "Iorsque le vent est violent, il balaye du sable, ce qui rend la marche très fatigante"

Sur le Platon, à l'endroit le plus éminent de la ville, se dresse une maison de pierre fort imposante, la "maison du roi", qui sert de résidence aux gouverneurs ${ }^{62}$ : c'est ici que loge le nouveau gouverneur anglais, Ralph Burton, assisté dans l'administration par un secrétaire bilingue et d'origine huguenotte, John Bruyère. Tout près de la "maison du roi", nous remarquons un moulin à vent ${ }^{63}$ et un second, à l'ouest, sur le terrain de la Commune ${ }^{64}$. A l'angle des rues Saint-Pierre et Notre-Dame, l'église paroissiale construite de pierre, "bien bâtie, grande et bien ornée; il y a entre autres choses remarquables, une chaire d'une sculpture des plus fines et des plus recherchées" ${ }^{65}$ : c'est un travail de Gilles Bolvin ${ }^{66}$; "le banc-d'œuvres, avec son dossier de vingt pieds de haut, est de toute beauté; il n'a pas son pareil en Amérique" ${ }^{\prime 67}$. Les Récollets sont curés de la paroisse depuis $1670^{68}$. Les affaires spirituelles du Gouvernement en général relèvent du grand-vicaire Joseph-François Perrault.

La rue Notre-Dame, qui donne sur le Platon, est la première rue parallèle au fleuve, c'est la rue importante de la ville. En nous dirigeant vers l'est, nous voyons tout de suite, à notre gauche, la maison de Louis-Joseph Godefroy de Tonnancour, ci-devant garde-magasin $\mathrm{du}$ Roi, proprietaire de six fiefs, "homme fort riche, d'une belle figure et de beaucoup d'esprit" ${ }^{69}$. En face de cette maison, s'étend la Place d'Armes, sur le côté sud de la rue. Tout près de là, du même

61. Voyage de Kalm en Amérique, op. cit., 65.

62. C'est la maison qui servira plus tard de caserne, puis, en 1860, de local au premier séminaire des Trois-Rivières. Voir Sulte, Mélanges historiques, XVIII: 56; XIX: 41s; abbé L. Richard, Histoire du Collège des Trois-Rivières. Première période, de 1860 à 1874 (Trois-Rivières, 1885), 41ss.

63. Vis-à-vis la rue Radisson (Sulte, op. cit., XVIII: 51s.; du temps de Sulte, cette rue portait le nom d'Alexandre).

64. Le moulin Laguerche (ibid.: 59ss.)

65. Franquet, op. cit., 16.

66. Sulte, op. cit., XIX: 83.

67. Ibid., 29. Cette église, qui datait de 1715, a été incendiée en 1908.

68. P. Odoric-M. Jouvet, o.f.m. Les Franciscains et le Canada. Aux Trois-Rivières (Paris, 1934), 204-212.

69. Franquet, op. cit., 16s. Cette maison, encore bien conservé, est occuṕe aujourd'hui par les Filles de Jésus. 
côté, un terrain de vingt toises sur lequel les Récollets ont construit un couvent en 1742 et une église de pierre en 1754: il ne s'y trouve que quatre ou cinq récollets ${ }^{70}$; le couvent tient lieu de presbytère paroissial: c'est dans le parloir de ce couvent que les habitants vont déposer leurs armes et prêter le serment de fidélité au nouveau roi, après la conquête. ${ }^{71}$ Plus à l'est, une poudrière "enveloppée d'une enceinte de pieux" ". Au nord, de l'autre côté de la rue, le couvent des Ursulines, reconstruit depuis 1753 ; il y a là une vingtaine de religieuses: "elles sont chargées de l'hôpital militaire et tiennent des jeunes filles en pension"73. Le reste de la ville est composé généralement de maisons "en bois, à un seul étage, assez bien bâties et très éloignées les unes des autres"74.

Outre l'école des filles, dirigée par les Ursulines, il y a aussi, semble-t-il, une école gratuite pour garçons, tenue par les Récollets $^{75}$; il est possible que Denys Ponsant, qui habite la ville et figure en 1761 comme maître d'école, se consacre en ce moment à l'instruction des enfants ${ }^{75 \mathrm{a}}$. Trois notaires exercent leurs fonctions aux Trois-Rivières: Louis Pillard, Jean Le Proust et Paul Diel ${ }^{76}$. On ne connaît qu'un seul chirurgien: Charles Alavoine ${ }^{77}$. Trois marchands viennent d'obtenir un permis pour faire un "commerce fixe": LouisGodefroy de Tonnancour, Desbarras et Laframboise ${ }^{78}$. Comme l'avait déjà noté Franquet, "les habitants y sont plus adonnés à la culture

70. Jouve, loc. cit.

71. Ibid.,

72. Franquet, op. cit., 112; Sulte, Mélanges, XVIII: 45s.

73. Franquet, op. cit., 16; Les Ursulines des Trois-Rivières, (4 vol. Québec, 1863), IV: $478 \mathrm{ss}$.

74. Kalm, op. cit., 65 .

75. Sulte, op. cit., XIX: 21; Jouve, op. cit., 283.

75a. APJTR, Insinuations October 1761 to June 1762, f. 14v. Il est dans cette ville en octobre 1760: RAPQ (1946-1947), 8.

76. J.-E. Roy, Histoire du notariat... II: 11 et 14.

77. Dr M.-J. \& G. Ahern, Notes pour servir à l'histoire de la médecine dans le BasCanada depuis la fondation de Québec jusqu'au commencement du XIXe siècle (Québec, 1923), 5 .

78. RAPQ (1946-1947), 8. 
des terres qu'au commerce"'79; sur les 5,830 acres concédées, ils en exploitent 1,339 ; ils possèdent 80 chevaux, 210 bêtes à cornes et 50 moutons: ce qui est à peu près ce qu'on trouve dans une paroisse rurale moyenne ${ }^{80}$.

Trois-Rivières est surtout remarquable pour son industrie du canot: "c'est l'endroit de tout le Canada où on travaille le mieux les canots d'écorce dont on se sert pour voyager dans les pays d'en haut"; la main-d'œuvre est fournie par les femmes et les jeunes filles ${ }^{81}$; a l'époque de la conquête, il semble bien que l'industrie du canot ne soit guère florissante, puisqu'elle ne méritera qu'une simple mention dans le rapport de Burton ${ }^{82}$. Quant à la traite des pelleteries qui faisait jadis des Trois-Rivières une véritable foire internationale, elle n'amène plus que les nations errantes du Saint-Maurice ${ }^{83}$.

A sept milles au nord de la ville, derrière les fiefs que nous venons d'énumérer, s'étend la seigneurie de Saint-Maurice qui, avec l'ancien fief Saint-Étienne réuni au Domaine en 1737, constitue une seigneurie de quatre lieues de front sur la rivière Saint-Maurice par une profondeur d'environ deux lieues ${ }^{84}$; l'ancienne Compagnie qui en était la seigneuresse, a été dissoute lorsque le Roi, en 1741, s'empara de l'exploitation. Au village des Forges, où l'on parvient des Trois-Rivières par le "chemin des Forges" ou par la rivière, on travaille le fer depuis 1738; c'est un petit village ouvrier de 72 habitants, dont onze chefs de maison et 18 garçons; ils ont une chapelle de bois où le récollet Hyacinthe Amiot exerce son ministère ${ }^{85}$. L'ouvrage va bientôt reprendre dans les forges ${ }^{86}$.

Nous redescendons vers le fleuve, "passant à travers un pays

79. Franquet, op. cit., 109.

80. Doc. const., 76.

81. Franquet, op. cit., 17 et 109.

82. Burton n'en dit pas un mot dans son rapport de 1762; dans celui de 1763 (AC, B 21-1: 11), il se contente d'y faire allusion.

83. Franquet, op. cit., 110.

84. P.-G. Roy, Invent. des concessions..., II: 115-117; Bouchette, Description topographique..., 311-317.

85. RAPQ (1946-1947), 52s; Jouve, op. cit., 290.

86. Voir notre chapitre sur les Forges. 
assez élevé, sablonneux et à surface plane" ${ }^{\prime 87}$ pour reprendre aux TroisRivières le chemin du roi. Ce chemin du roi, qui passe tout près de la ville, sans y entrer ${ }^{88}$, nous conduit vers la Pointe-du-Lac. A l'ouest de la Commune, signalons, en passant, le Marquisat du Sablé qui n'est, malgré son titre pompeux, qu'une terre de dix arpents en roture, propriété de la famille François Chastelain ${ }^{89}$; entre le ruisseau du Moulin-à-Vent, appelé aussi Deuxième Rivière ${ }^{90}$, et la Troisième Rivière, nous traversons le fief des Jésuites, 14 arpents de front sur 25 de profondeur ${ }^{91}$. Au-delà de la Troisième Rivière, la seigneurie Vieuxpont, une lieue par six, propriété de la famille Godefroy de Normanville ${ }^{92}$; à partir de la Quatrième Rivière, située à une lieue de la capitale, la seigneurie Labadie qui ne mesure qu'un quart de lieue par une demie et dont Louis-Joseph Godefroy de Tonnancour est le seigneur ${ }^{93}$; à trois cents pas au-delà de la Cinquième Rivière, le fief Boucher, dix arpents par vingt, qui appartient à Louis Lemâ̂tré $^{94}$ et, enfin, la seigneurie Tonnancour ou Pointe-du-Lac, d'une lieue et quart de front sur le lac Saint-Pierre par deux lieues de profondeur, autre seigneurie de Louis-Joseph Godeiroy de Tonnancour ${ }^{95}$.

Tous ces fiefs font partie de la paroisse de la Pointe-du-Lac qui n'a cependant que 178 habitants et 34 maisons; sur 2,780 acres concédées, 280 seulement sont en culture; le bétail se répartit entre 20 chevaux, 50 bêtes à cornes et 12 moutons ${ }^{96}$. Il y a, en cet endroit, un

87. Kalm, op. cit., 66.

88. Des bords du Saint-Maurice, il passait au nord du Haut-Boc, puis venait rejoindre ce qui est aujourd'hui la rue Royale et coupait la rue des Forges; il n'entrait donc pas dans la ville de 1760 (Sulte, Mélanges, XVIII: 68ss.)

89. P.-G. Roy, Invent. des concessions, I: 289-291.

90. De la rue St-Antoine à la Pointe-du-Lac, on compte cinq rivières.

91. Pièces et documents relatifs à la tenure seigneuriale, (Québec, 1852) II: huitime partie, 2 et 18; Sulte, op. cit., 16s.

92. P.-G. Roy, Invent. des concessions..., I: 286-289.

93. P.-G. Roy, Inventaire des concessions..., II: 125-127.

94. Ibid.: 50-53.

95. Ibid., II: 44-40; Dugré, La Pointe-du-Lac, 17; Bouchette, Description topagraphique..., 304s.

96. RAPQ (1946-1947), 19s, 52s; Doc. const., 76. 
village d'Algonquins: "J'apperçus, écrivait Franquet en 1752, des maisons bâties uniformément et assujetties à des alignements, il y en avait déja neuf: surpris de cette régularité, mon dit sieur de Tonnancour me dit que c'était luy qui les faisait construire à ses dépens et sur un terrain à lui appartenant pour y réfugier des sauvages errans et vagabonds... Indépendamment des sauvages algonquins, il compte aussi d'autres Têtes de Boules et Montagnais"'97. Lors du recensement, Louis-Michel Guay figure comme curé, mais il va partir bientôt pour Sainte-Anne-de-la-Pérade, laissant la place au récollet Dominique Pétrimoulx qui viendra périodiquement y faire le service religieux; la paroisse a son église et son presbytère ${ }^{98}$.

A l'ouest de la seigneurie de Tonnancour, s'étend celle de Gastineau, qui mesure trois quarts de lieue par une lieue; elle est la propriété de Louis-Joseph Gastineau qui réside dans sa seigneurie de Sainte-Marie, à Sainte-Anne ${ }^{99}$. Dans les profondeurs, le fief Robert, qui est la prolongation de la seigneurie Gastineau et couvre trois quarts de lieue par quatre, appartient à Marie-Josephte Gastineau, sœur du seigneur voisin ${ }^{1}$. Sur le lac Saint-Pierre, en allant toujours en amont, nous entrons dans la seigneurie Grosbois-Est, dite aussi Grande-Rivière-Yamachiche, où les Lesieur sont seigneurs d'un fief de trois quarts de lieue par deux lieues; la grande Rivière Yamachiche vient se déverser dans le lac, tout près de la frontière occidentale de la seigneurie ${ }^{2}$. Grosbois-Ouest, qui vient ensuite, a à peu près les mêmes dimensions; le seigneur des lieux, Louis Boucher de Grandpré, est absent à la Nouvelle-Orléans ${ }^{3}$. Derrière les profondeurs de ces deux fiefs Grosbois, la seigneurie Dumontier couvre une lieue et demie par trois, traversée en diagonale par la rivière du Loup:

97. Franquet, Voyages, 23. Burton, en 1762, fait allusion à cet établissement, mais ne donne pas de chiffres (Doc. const., 75).

98. Dugré, op. cit., 34-36, 39.

99. P.-G. Roy, Invent. des concessions, II: 253-255; R. Bellemare, Les Bases de l'histoire d'Yamachiche 1703-1903, (s.l.n.d.), 50-58, 238s.; Bouchette, op. cit., 303s.

1. Ibid.

2. P.-G. Roy, Invent. des concessions, II: 270-274; Bellemare, op. cit., 44-49, 236-238; Bouchette, op. cit., 302s. Le front est de trois quarts de lieue, plus sept arpents.

3. P.-G. Roy, Invent. des concessions, II: 265-270; Bellemare, op. cit., 37-43, $235 \mathrm{~s}$. Le front mesure trois quarts de lieue, moins sept arpents. 
les quatre filles de François Dumontier, premier concessionnaire, sont les seigneuresses de ce fief ${ }^{4}$. Avec ces cinq fiefs, on a la paroisse d'Yamachiche, dont Jacques-Maxime Chefdeville de la Garenne est le curé résidant; l'église de pierre est située près de l'embouchure de la grande Rivière Yamachiche, sur la rive occidentale ${ }^{5}$. La paroisse compte 103 maisons et 566 habitants, qui en fait la paroisse la plus importante du Gouvernement, immédiatement après les TroisRivières; le recensement donne 1,800 acres en valeur sur 9,800 concédées, 90 chevaux, 250 bêtes à cornes et 70 moutons $^{6}$.

La seigneurie de Grandpré, qui avoisine Grosbois-Ouest sur le lac Saint-Pierre, mesure une lieue par trois; elle appartient au même seigneur, Louis Boucher de Grandpré ${ }^{7}$. En amont, les Ursulines des Trois-Rivières sont seigneuresses de Rivière-du-Loup, fief d'une lieue par quatre, traversé presque en son milieu par la rivière du Loup; "il surpasse peut-être en valeur toutes les propriétés d'une pareille étendue": le sol, en plusieurs endroits, est "d'une belle marne jaune très-fertile, qui produit d'abondantes récoltes de grain, et en général de toutes les productions du pays':8. Le fief voisin, Saint-Jean ou Maskinongé-Est, appartient aussi aux mêmes Ursulines: à sa superficie de trois quarts de lieue par deux lieues s'ajoute une augmentation de trois lieues ${ }^{9}$. La paroisse de Rivière-du-Loup couvre ces fiefs: elle a 414 habitants, dont 8 Acadiens, logés dans 116 maisons; sur 6,200 acres concédées, 1,200 ont été mises en valeur; on possède 90 chevaux, 160 bêtes à cornes et 20 moutons, et l'on cultive surtout du blé et de l'avoine ${ }^{10}$. Les paroissiens ont leur église, ainsi que leur curé, Médard Pétrimoulx, aidé dans son ministère par son frère, 239s.

4. P.-G. Roy, Invent. des concessions..., IV: 203-207; Bellemare, op. cit., 63-66,

5. Bellemare, op. cit., 184; voir le plan vis-à-vis, p. 235.

6. RAPQ (1946-1947), 15-18, 52s.; Doc. const., 76.

7. P.-G. Roy, Invent. des concessions..., IV: 117-119; Bouchette, op. cit., 301s.

8. P.-G. Roy, Invent. des concessions..., III: 61-64; Sulte, Mélanges, X 5-65; F.-J. Audet, Le comté de Maskinongé 1853-1867 (Trois-Rivières, 1934), 20; Bouchette, op. cit., 299-301.

9. P.-G. Roy, Invent. des concessions..., IV; 163-165; Audet, op. cit., 22; Bouchette, op. cit., $298 \mathrm{~s}$.

10. RAPQ (1946-1947), 11-15, 52s.; Doc. const., 76. 
le récollet Dominique Pétrimoul ${ }^{11}$. Un chirurgien, François-Joseph Rimbaud, réside temporairement dans cette paroisse, à l'époque du recensement ${ }^{12}$.

A l'ouest de la seigneurie Saint-Jean, toujours sur le lac SaintPierre, la seigneurie de Maskinongé étend son front de deux lieues et demie; sa profondeur irrégulière se rend jusqu'à une lieue et demie dans les terres: elle appartient à Geneviève Sicard de Carufel, veuve de Jean-François Baril-Duchesny ${ }^{12^{\mathrm{a}}}$. Derrière cette seigneurie, la veuve Duchesny en possède une autre, de deux lieues carrées, la seigneurie dite Carufel ou l'Ormière ${ }^{13}$. Au bout des profondeurs de cette dernière, Charles-François Tarieu de Lanaudière possède la seigneurie du lac Maskinongé: on avait donné à cette seigneurie un front de deux lieues et une profondeur qui pût englober le lac Maskinongé; or, comme le lac se trouve beaucoup plus à l'ouest qu'on ne le croyait, le domaine de Lanaudière prend une forme très irrégulière ${ }^{14}$. "C'est écrit Bouchette, une belle étendue de terrain, d'un sol fort et fertile, et bien boisé de hêtre, d'érable, de bouleau, de pin, et de quelques chênes... Le paysage d'alentour possède pluśieurs beautés naturelles dans le genre sauvage et sublime, offrant un amphithéâtre d'éminences et de vastes collines, couronnées par derrière par la magnifique chaîne de montagnes qui se prolonge à l'ouest depuis Québec, et plusieurs autres des traits hardis qui forment un pays romantique"15. Enfin, entre la seigneurie de Carufel et la limite du Gouvernement des Trois-Rivières, mentionnons la seigneurie Dusablé, une lieue par trois, dont la seigneuresse est Marie-Josephte Drouet de Richerville, veuve de Louis-Adrien Dandonneau du Sable ${ }^{16}$.

11. Jouve, op. cit., 297; Sulte, Mélanges, X: 43.

12. RAPQ (1946-1947), 14; Douville, Chirurgiens, loc. cit., 122-124.

12a. P.-G. Roy, Invent. des concessions..., III: 88-95; Audet, op. cit., 17-19; Bouchette, op. cit., 294-296.

13. P.-G. Roy, Invent. des concessions..., IV: 184-188; Audet, op. cit., 22s.; Bouchette, op. cit., 296s.

14. P.-G. Roy, Invent. des concessions..., III: 268-272; Audet, op. cit., 20s. Tarieu de Lanaudière est déjà propriétaire de la moitié occidentale de Sainte-Anne; il vient de passer en France.

15. Bouchette, Description topographique... 297s.

16. P.-G. Roy, Invent. des concessions..., V: 54-56; Audet, op. cit., 19; Bouchette, op. cit., $296 \mathrm{~s}$. 
Ces derniers fiefs sont rattachés à la paroisse de Maskinongé qui compte 331 personnes et 67 maisons; les habitants possèdent 50 chevaux, 150 bêtes à cornes et 25 moutons; ils cultivent du blé, de l'avoine et des pois: sur les 4,250 acres concédées, 550 sont mises en valeur ${ }^{17}$. L'église qui date de 1739 , est sans curé résidant, elle est desservie par Médard Pétrimoulx, aidé de son frère le récollet ${ }^{18}$. La paroisse jouit des services d'un notaire, Pierre-François Rigault ${ }^{19}$.

Nous traversons ensuite le lac Saint-Pierre pour reprendre notre inventaire sur la rive sud, où nous trouvons, à la limite occidentale du Gouvernement, la seigneurie La Vallière ou Yamaska qui, dans son front d'une demi-lieue, comprend l'embouchure de la rivière Yamaska; sa profondeur est de trois lieues; Louis-Joseph Godefroy de Tonnancour en est le propriétaire ${ }^{20}$. Autour de la baie d'Yamaska, "le terrain est bas et marécageux, mais à peu de distance il y a plusieurs vastes et excellentes prairies; en tirant plus vers le fond le sol est gras et très-fertile; il est composé d'une bonne marne jaune, et d'un belle argile entremêlée de terre légère, qui forment de trèsbonne terre labourable pour le grain de toute espèce, et quelques terrains très propres à la culture du chanvre et $d u$ lin... La rivière Yamaska procure une navigation intérieure assez importante pour les batteaux et les radeaux"; il n'y a point de pont pour la franchir ${ }^{21}$. La paroisse Yamaska, contient 483 habitants et 117 maisons; ils exploitent 1,300 acres sur les 9,300 concédées; le recensement indique 120 chevaux, 380 bêtes à cornes et 150 moutons: c'est la paroisse la plus riche du Gouvernement par la quantité des animaux et par celle, en particulier, des bêtes à cornes ${ }^{22}$. Le curé de cette paroisse prospère est Joseph-Basile Parent ${ }^{23}$.

17. RAPQ (1946-1947), 9-11, 52s.; Doc. const., 76.

18. Hermann Plante, Saint-Justin, 16 et 21. Jouve, op. cit., 297.

19. J.-E. Roy, Histoire du notariat, II: 11.

20. P.-G. Roy, Invent. des concessions..., III: 261-266; P.-G. Roy, La famille Godefroy de Tonnancour. (Lévis, 1904), 51-57.

21. Bouchette, op. cit., 327-329.

22. RAPQ (1946-1947), 47-50, 52s (donne, par erreur, 750 moutons au lieu de 150); Doc. const., 76.

23. Thomas-M. Charland, O.P., Histoire de Saint-François-du-Lac. (Ottawa, 1942), 202. 
A l'est, nous pénétrons dans la seigneurie de la Rivière-SaintFrançois qui couvre une lieue et demie le long du lac; à une lieue de la rive, sa profondeur devient d'une largeur de deux lieues par une; Joseph Crevier est le seigneur de ce domaine ${ }^{24}$. En aval, sur le lac, la famille de Jean-Baptiste Jutras-Desrosiers possède une seigneurie d'une lieue carrée, dite Lussodière ${ }^{25}$. Derrière Saint-François, s'étend la seigneurie de Pierreville sur un front d'une lieue et demie et sur une profondeur d'une seule, traversée en son milieu par la rivière SaintFrançois; elle appartient à Joseph $\mathrm{Hertel}^{26}$. Enfin, derrière les seigneuries Pierreville, Saint-François et Bourgmarie ${ }^{27}$, on a RivièreDeguire, seigneurie de deux lieues carrées qui est à Joseph DeguireDesrosiers ${ }^{28}$. Ces quatre seigneuries constituent la paroisse de SaintFrançois-du-Lac qui compte 319 habitants et 59 maisons; le bétail se répartit entre 60 chevaux, 180 bêtes à cornes et 40 moutons; on cultive surtout du blé et du blé d'Inde; 1,100 acres sur 2,600 sont mises en valeur ${ }^{29}$. L'église, richement décorée, est terminée depuis 1739; Jean-Baptiste Dugast y exerce les fonctions curiales depuis déjà 1714; la paroisse a son notaire, Antoine Robin ${ }^{30}$.

C'est dans les profondeurs de la seigneurie de Saint-François, à deux lieues de l'embouchure de la rivière, sur la rive est, que sont installés les Abénaquis qui vivaient autrefois sur la Chaudière; le seigneur de Saint-François leur concéda en 1700 un terrain de deux lieues et demie de front par une demi-lieue de profondeur, puis un quart de lieue de plus le long de la rivière, à condition que le tout revienne à la seigneurie si les sauvages désertent la mission ${ }^{31}$. Du village Saint-François à cette mission abénaquise, il n'y a qu'une lieue; Franquet, qui l'a visitée en 1752 , nous dit qu'il y a compté

24. P.-G. Roy, Invent. des concessions..., V: 114-121; Bouchette, op. cit., 329335; Charland, op. cit., 89-92.

25. P.-G. Roy, Invent. des concessions..., III: 72-76; Bouchette, op. cit., 335s.

26. P.-G. Roy, Invent. des concessions..., III: 247-249; Bouchette, op. cit., 336s.

27. Partie d'une seigneurie du Gouvernement de Montréal.

28. P.-G. Roy, Invent. des concessions..., V: 78-81; Bouchette, op. cit., 337s.

29. RAPQ (1946-1947), 44-46, 52s.; Doc. const., 76.

30. Charland, op. cit., 175-202; J.-E. Roy, Histoire du notariat, II: 14.

31. P.-G. Roy, Invent. des concessions..., V: 117; Charland, op. cit., 72ss; J.-A. Maurault, Histoire des Abenaquis depuis 1605 jusqu'à nos jours (Sorel, 1866), 277ss . 
"51 cabannes, figure carrée, construites en bois équarry comme celles du saut St. Louis et du lac des deux montagnes, néanmoins couvertes de planches et d'écorces, en figure de tourelle, et douze autres bâties à la française"'32. C'est là que le major Robert Rogers alla faire sa terrible descente d'octobre 1759, afin de punir les Abénaquis "de leur perfidie dans l'arrestation de deux officiers anglais que leur avait envoyés le général Amherst pour leur proposer de rester neutres, en même temps que pour venger toutes les captures et tous les massacres" dont les sauvages s'étaient rendus coupables ${ }^{33}$. Avec environ deux cents hommes, le 4 octobre, "une demi-heure avant le lever du soleil, il fondait sur la place, à la fois par la droite, la gauche et le centre, et surprenait tous ses habitants dans le plus profond sommeil. Officiers et soldats attaquèrent avec tant de promptitude que les Sauvages n'eurent pas le temps de se ressaisir et de prendre les armes pour se défendre... Un peu après le lever du soleil, ils mirent le feu à toutes les maisons, à l'exception de trois, où se trouvait du blé qu'ils se réservèrent... Ils incendièrent aussi le couvent des Jésuites et l'église de la mission, après en avoir pillé les objets précieux et profané les Saintes Espèces... Vers 7 hrs. [ sic ] toute l'affaire était terminée. Une vingtaine de femmes et d'enfants avaient eté faits prisonniers. Rogers en relâcha quinze, pour n'amener que deux garçons et trois filles" ${ }^{34}$. Cette mission de Saint-François est le seul coin du Gouvernement des Trois-Rivières à avoir subi les effets dévastateurs de la conquête. En 1760 , elle commence à peine à se relever de ses ruines: les Abénaquis, au nombre de 700, ont comme missionnaire le jésuite Pierre-Antoine-Basile Roubaud; le curé de Saint-François s'occupe aussi de la mission ${ }^{35}$.

A l'est de la seigneurie de Saint-François, celle de la Baie-SaintAntoine ou Baie-du-Febvre forme un carré de deux lieues avec front sur le lac; elle a, pour seigneur en titre, Joseph Lefebvre ${ }^{36}$. Dans les

32. Franquet, Voyages, 94s.

33. Charland, op. cit., 108.

34. Ibid., 108-115; Sulte, Histoire de Saint-François-du-Lac, 103s.; Maurault, op. cit., 488-496; voir aussi le roman de Kenneth Roberts, Northwest Passage (1937).

35. Charland, op. cit., 195-202; Maurault, op. cit., 629.

36. P.-G. Roy, Invent. des concessions..., III: 249-258; Bouchette, Description topographique..., 339-341; Bellemare, Histoire de la Baie Saint-Antoine dite Baie-duFebvre 1683-1918 (Montréal, 1911), 418. 
profondeurs, on a concédé récemment la seigneurie de Courval, deux lieues par trois, à Louis-Pierre Poulin-Cressé, aide-constructeur de vaisseaux, fils du seigneur de $\mathrm{Nicolet}^{37}$. La paroisse, BaieSaint-Antoine, compte 279 habitants et 57 maisons, 1,000 acres en valeur sur 6,000 de concédées, 50 chevaux, 200 bêtes à cornes et 80 moutons; la culture est surtout en blé et en pois ${ }^{38}$. L'église de pierre a été terminée tout juste avant la conquête; c'est le curé de Nicolet, Louis-Marie-Ferdinand Brassard, qui vient y assurer le service religieux ${ }^{39}$.

Au-delà de la limite orientale de Baie-du-Febvre, commence la seigneurie de Nicolet, de deux lieues par cinq, dont Claude PoulinCressé est le seigneur ${ }^{40}$. A l'embouchure de la rivière Nicolet, il y a la petite île Moras, qui mesure à peu près une demi-lieue par un quart, constituée en seigneurie depuis 1672: elle appartient à Louis Beaubien $^{41}$. Trois autres petites seigneuries s'échelonnent ensuite le long du lac et sont toutes le domaine du même seigneur, Lou isJoseph Godefroy de Tonnancour: Roquetaillade, une demi-lieue par trois; Godefroy ou Linctot, trois quarts de lieue par trois; l'île Marie, petit fief de vingt arpents, à l'entrée de la rivière Godefroy ${ }^{42}$. Nicolet sert de paroisse a toutes ces seigneuries: le recensement lui donne 268 habitants et 59 maisons, 1,200 acres en valeur sur 9,200, 60 chevaux, 210 bêtes à cornes et 80 moutons $^{43}$. L'église de pierre, qui date de 1734, est située sur la rive gauche de la Nicolet et regarde

37. P.-G. Roy, Invent. des concessions... V: 85-88; Bellemare, Histoire de Nicolet 1669-1924 (Arthabaska, 1924), 120-122; Bouchette, op. cit., 341. La concession ne date que de 1754 .

38. RAPQ (1946-1947), 42-44, 52s.; Doc. const., 76.

39. Bellemare, Histoire de la Baie-du-F ebvre, 59ss; cette paroisse restera desserte jusqu'en 1786.

40. P.-G. Roy, Invent. des concessions..., II: 238-243; Bellemare, Histoire de Nicolet, 117-124; Bouchette, op. cit., 342-346. Ce seigneur est le père de Louis-Pierre, seigneur de Courval, et de Joseph-Claude, directeur des Forges.

41. P.-G. Roy, Invent. des concessions..., II: 236-238; Bellemare, op. cit., 75; Bouchette, op. cit., 343.

42. P.-G. Roy, Invent. des concessions..., I: 151-154; II: 53; III: 145-149; Bouchette, op. cit., 346s.

43. RAPQ (1946-1947), 39-42, 52s.; Doc. const., 76. 
"le haut de la rivière": Louis-Marie-Ferdinand Brassard y poursuit son long terme d'un demi-siècle ${ }^{44}$.

La seigneurie de Bécancour ou Rivière-Puante qui avoisine Godefroy sur le fleuve, s'étend sur deux lieues et demi carrées; concédée depuis 1647, elle appartient aujourd'hui à Joseph-Michel Le Gardeur de Croizille et de Montesson; elle inclut le lac Saint-Paul: c'est, suivant la description de Bouchette, "un élargissement d'environ quatre milles et demi de longueur, et d'un demi-mille de largeur... Ses bords offrent un paysage parfait relevé, par presque toute espèce de points de vue charmans" ${ }^{45}$. A partir de la rive droite de la rivière Bécancour, on a la petite seigneurie Dutort, un quart de lieue de front par trois de profondeur, qui appartient, semble-t-il, à Hyacinthe Godefroy de Linctot ${ }^{46}$. Ces deux dernières seigneuries forment la paroisse de Bécancour où l'on trouve 353 habitants, dont 39 Acadiens, logés dans 73 maisons; sur les 3,400 acres concédées, 400 seulement sont mises en valeur; on dispose de 40 chevaux, 200 bêtes à cornes et 20 moutons $^{47}$. C'est un jésuite, le père Simon-Pierre Gonnon, qui y exerce les fonctions curiales ${ }^{48}$. Comme à Saint-François, il y a à Bécancour une réserve d'Abénaquis pour laquelle, en 1708, le baron de Portneuf avait prêté une superficie de six milles, dans la partie ouest de la seigneurie, derrière les concessions des habitants; de cinq cents qu'ils étaient dans les débuts, les Abénaquis ne sont plus que trois cents environ ${ }^{49}$. Franquet, qui les a visités en 1752 , écrit que le village " $n$ 'est pas considérable, il n'a que 19 cabanes, toutes de figure carrée longue, construites et couvertes comme celles du village St-François" ${ }^{50}$. Les sauvages n'ont pas reconstruit leur église que le feu a détruite en 1757 et ils fréquentent l'église paroissiale de Bécancour ${ }^{51}$.

44. Bellemare, Histoire de Nicolet, 364 et 368. Brassard fut curé de Nicolet de 1750 à 1800.

45. P.-G. Roy, Invent. des concessions..., I: 251-257; Bouchette, op. cit., 347-351.

46. P.-G. Roy, Invent. des concessions..., I: 150s.; P.-G. Roy, La famille Godefroy de Tonnancour, $27 \mathrm{~s}$.

47. RAPQ (1946-1947), 36-39, 52s.; Doc. const., 76.

48. J.-A. Maurault, Histoire des Abénaquis, 5048.

49. Ibid., 284-298.

50. Franquet, Voyages, 99.

51. Maurault, loc. cit. 
En aval de Bécancour, la famille Hertel de Cournoyer possède la seigneurie de Cournoyer, carré de deux lieues sur le fleuve ${ }^{52}$; plus bas, la seigneurie de Gentilly, qui mesure deux lieues et demie par deux, a pour seigneur en titre, François Poisson: soixante co-seigneurs se partagent le troisième quart de cette seigneurie ${ }^{53}$. L'un et l'autre de ces fiefs sont rattachés à la paroisse de Gentilly: 164 habitants, dont 25 Acadiens; 26 maisons, 200 acres en valeur sur les 5,800 concédées, 24 chevaux, 60 bêtes à cornes et 17 moutons $^{54}$. La paroisse n'a ni église ni curé, c'est Morisseau-Boismorel, curé de Champlain, qui vient officier dans une maison privée ${ }^{55}$.

Enfin, nous arrivons à la dernière seigneurie du Gouvernement des Trois-Rivières, sur la rive sud, à la seigneurie de Lévrard, deux lieues par quatre, qui appartient à Charles Lévrard ${ }^{56}$. SaintPierre-les-Becquets, qui en est la paroisse, compte 250 habitants, dont 13 Acadiens; on note, dans le recensement, 41 maisons, 400 acres en valeur sur les 3,900 concédées, 35 chevaux, 120 bêtes à cornes et 30 moutons $^{57}$. La chapelle, bâtie en 1731, est desservie par le curé de Deschaillons, Jean-Baptiste Gatien ${ }^{58}$.

Cette rive sud que nous avons parcourue, présente à peu près partout les mêmes caractéristiques depuis la seigneurie d'Yamaska à celle de Lévrard. Bouchette remarque, en général, que le front de ces seigneuries, sur le fleuve, est bas, marécageux et pauvre et qu'à mesure qu'on avance vers l'intérieur, le sol s'élève peu à peu et consiste surtout d'argile grasse et de bonne terre noire; la grande abondance de petits cours d'eaux ajoute à sa fertilité; le bois de construction est cependant d'une qualité qui laisse à désirer. C'est à partir

52. P.-G. Roy, Invent. des concessions..., I: 243s.; IV: 98-102; Bouchette, op. cit., 351s.

53. P.-G. Roy, Invent. des concessions..., I: 245-250; Bouchette, op. cit., 352s.; Lucien Dubois, Histoire de la paroisse de Gentilly (1935) 38s.

54. RAPQ (1946-1947), 35s., 52s.; Doc. const., 76.

55. Dubois, op. cit., 83 et 90.

56. P.-G. Roy, Invent. des concessions..., III: 234-238; Bouchette, op. cit., 353s.

57. RAPQ (1946-1947), 33s., 52s.; Doc. const., 76.

58. Félix-Lanzo Lemay, Monographie de Saint-Jean-Baptiste de Deschaillons (Québec, 1934), 232. Dubois écrit que Saint-Pierre eut une "église" avant 1756 (op. cit., 91). 


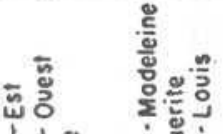

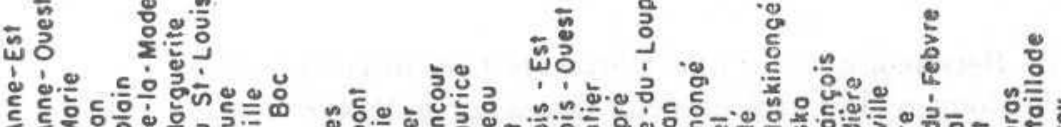

安

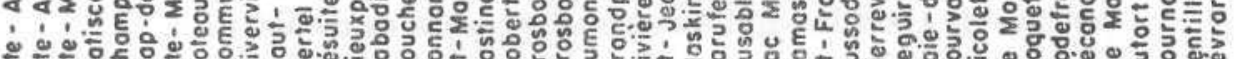

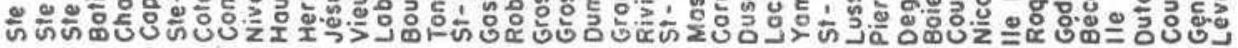

- NM⿻上ดN

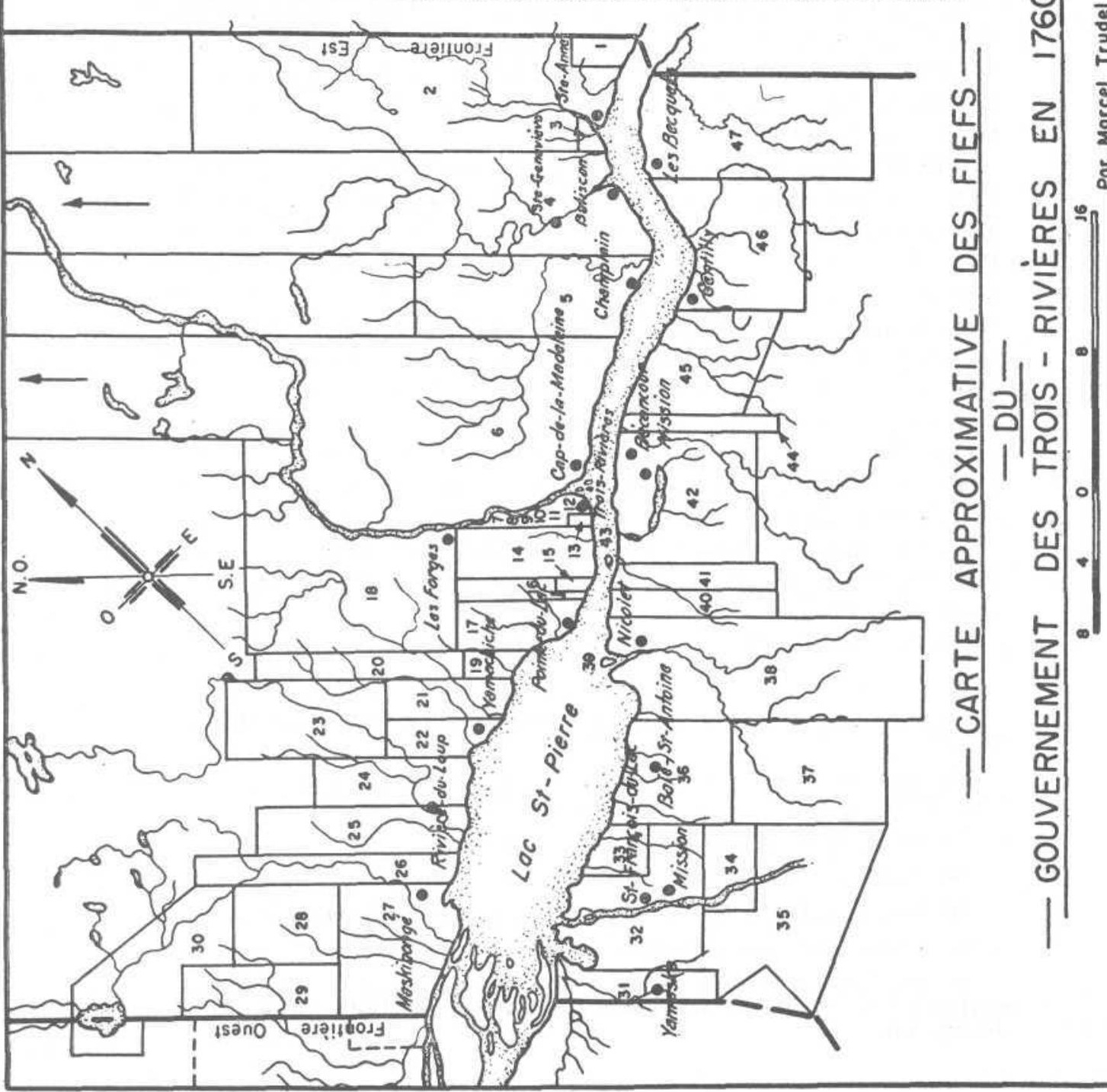


de Gentilly que la rive du fleuve "prend un caractère différent, elle est haute et escarpée, et de là le terrain s'abaisse par degré vers le fond"; en somme, c'est déjà la rive sud telle qu'elle se présente généralement, en aval, dans le Gouvernement de Québec ${ }^{59}$.

Nous pouvons donc maintenant essayer de nous faire une idée plus précise du Gouvernement des Troìs-Rivières, au moment où il passe sous le régime militaire. Comme dans les deux Gouvernements voisins, les deux rives sont couvertes de seigneuries d'une frontière à l'autre. Sur la rive nord, de Sainte-Anne à la rivière Saint-Maurice, le rang des seigneuries reste simple, mais par contre les principaux fiefs s'étendent bien avant dans les terres: c'est ainsi que Batiscan et le Cap-de-la-Madeleine pénètrent jusqu'à vingt lieues dans les profondeurs et constituent les plus grandes seigneuries du Gouververnement des Trois-Rivières et les plus profondes du Canada. De la rivière Saint-Maurice à la frontière occidentale du gouvernement, le rang des fiefs est double et il devient même triple près de la frontière du Gouvernement de Montréal. Triple, il l'est encore sur la rive sud, toujours près de la frontière de Montréal, puis, en descendant en aval, le rang devient double et, finalement, comme sur la rive nord, nous n'avons plus qu'un rang simple, avec cette différence considérable que les seigneuries de la rive sud ne dépassent pas, en cette partie, une profondeur de quatre lieues.

Le Gouvernement groupe cinquante et un fiefs, donnant un total de 93,140 acres concédées. La rive nord compte à elle seule trente-quatre fiefs dans lesquels on a concédé 47,110 acres. Il y a lieu de s'étonner que la concession des terres n'y soit pas plus avancée: la rive nord est arrosée de trois cours d'eau importants, la SainteAnne, la Batiscan et le Saint-Maurice, qui, malgré leurs rapides, auraient $d \hat{~}$ faciliter la pénétration à l'intérieur du pays. Les seigneuries de Batiscan et du Cap-de-la-Madeleine qui mesurent, chacune, deux lieues par vingt, n'ont de concédées que 9,633 acres, alors que, dans la seule seigneurie d'Yamaska qui ne mesure qu'une demi-lieue par

59. Bouchette, op. cit., 327-354. 
trois, nous en trouvons 9,300. Certes, le sol du Cap-de-la-Madeleine est palıvre, mais celui de Batiscan s'avère très fertile.

Les monopoles de seigneuries sont plus rares ici que dans les Gouvernements voisins, même si l'on tient compte du fait que le Gouvernement des Trois-Rivières n'a que 51 fiefs à opposer aux 96 de Montréal et aux 145 de Québec. Nous ne trouvons ici qu'un seul grand propriétaire terrien, la Compagnie de Jésus qui détient les deux plus profondes seigneuries de tout le Canada (Batiscan et Capde-la-Madeleine), outre quatre petits fiefs de peu d'importance: l'île Saint-Christophe, Pachirini, le Coteau Saint-Louis et le fief des Jésuites et de Saint-Paul. Le plus important propriétaire qui vienne ensuite est Tarieu de Lanaudière: il possède près de quatre lieues par huit. Il y a bien encore Louis-Joseph Godefroy de Tonnancour qui, avec ses six fiefs, prend figure de grand propriétaire, mais le nombre de ces fiefs ne doit pas nous faire oublier que le plus large ne dépasse pas une lieue et quart et qu'aucun n'avance plus de trois lieues dans les terres; si on les groupait tous, on n'obtiendrait tout au plus qu'un front de trois lieues et quart et une profondeur de trois lieues, seigneurie qui ressemblerait d'assez près à la seigneurie de Bécancour. Les autres propriétaires importants ont été servis assez modestement: les Ursulines, la veuve Duchesny et Boucher de Grandpré ont, chacun, à peu près un domaine total de deux lieues par trois.

La population globale de ce Gouvernement est de 5,871 habitants, chiffre assez pauvre auprès des 27,771 habitants du Gouvernement de Montréal et des 35,248 de celui de Québec ${ }^{60}$. Cette population compte très peu d'Acadiens, à peine 85: nous en avons rencontré huit à Rivière-du-Loup; les autres se sont retirés, sur la rive sud, près de la frontière orientale du Gouvernement: à Bécancour, à Gentilly et à Saint-Pierre-les-Becquets, comme s'ils craignaient de s'approcher des centres ${ }^{61}$; d'ailleurs, la plupart de ces Acadiens vont bientôt quitter les lieux, comme l'indiquera le recensement de $\mathbf{1 7 6 2}$. A cette population, il faut ajouter les mille Abénaquis des missions de Saint-François et de Bécancour et un nombre insignifiant d'Algonquins qui vivent à la Pointe-du-Lac.

60. Seuls les chiffres des Trois-Rivières sont de 1760, ceux de Montréal sont de 1765 et ceux de Québec, de 1762, mais la comparaison reste toujours légitime.

61. Pour deviner les craintes des Acadiens, il suffit de se rappeler les articles 38 et 39 de la Capitulation de Montréal: Doc. const., 17. 
Le Gouvernement comprend 18 paroisses et 2 missions. Si nous les groupons dans l'ordre d'importance numérique, nous avons le tableau suivant:

Mission abénaquise de St-François . . . . . . 700

Trois-Rivières ...................... 586

Yamachiche,$\ldots \ldots \ldots \ldots \ldots \ldots \ldots \ldots \ldots 6$

Sainte-Anne.................... 528

Sainte-Geneviève .................. 515

Yamaska........................ 483

Rivière-du-Loup . . . . . . . . . . . . . . . . . 414

Bécancour....................... 353

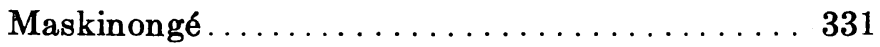

Saint-François-du-Lac . . . . . . . . . . . . . 319

Mịssion abénaquise de Bécancour.......... 300

Baie-du-Febvre . . . . . . . . . . . . . . 279

Nicolet...................... 268

Saint-Pierre-les-Becquets .............. 250

Champlain ...................... 216

Batiscan ........................ 189

Pointe-du-Lac . . . . . . . . . . . . . . . 178

Gentilly ....................... 164

Cap-de-la-Madeleine . . . . . . . . . . . . . 160

Les Forges................... 72

Trois-Rivières, pour sauvegarder son titre de capitale, vient en tête des paroisses, suivie tout de même d'assez près par Yamachiche. Par simple curiosité, comparons la population de cette capitale avec quelques paroisses des Gouvernements voisins:

Québec..................... 8,967

Montréal ................... 5,733

Sorel...................... 1,564

Charlesbourg .................

St-Thomas (Montmagny) ............. 1,152

Berthier-en-haut... .............. 1,007

Si l'on veut pousser plus loin la comparaison, on peut ajouter que la population des Trois-Rivières est encore dépassée par celle 
de paroisses comme Saint-Vincent-de-Paul, Varennes, Repentigny, Sainte-Rose, Pointe-Claire, Boucherville, Beauport, Cap-Sante, Saint-Charles-de-Bellechasse, Saint-Vallier, Cap Saint-Ignace, L'Islet, Kamouraska et Rivière-Ouelle, pour ne citer que celles-là. Bref, des 114 établissements de tout le Canada, la ville des Trois-Rivières n'arrive qu'au trentième rang pour la population.

Le chiffre de la population nous a donné un aperçu de l'importance respective des paroisses du Gouvernement, le nombre des maisons peut aussi nous en donner un autre par le tableau suivant:

Yamaska.................... 117

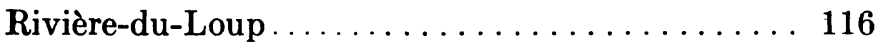

Trois-Rivières . . . . . . . . . . . . . 110

Yamachiche......................... 103

Ste-Anne . . . . . . . . . . . . . . . . . . . 101

Ste-Geneviève . . . . . . . . . . . . . . . . . 100

Bécancour .................... 73

Maskinongé................... 67

Nicolet....................... 59

St-François-du-Lac . . . . . . . . . . . . . 59

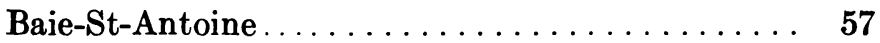

Champlain ..................... 50

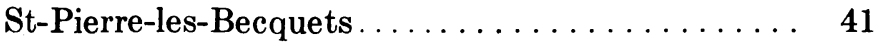

Batiscan ...................... 38

La Pointe-du-Lac . . . . . . . . . . . . . . . . . . . . 34

Cap-de-la-Madeleine .................. 33

Gentilly ................... 26

Trois-Rivières, qui arrive au premier rang des paroisses pour la population, n'est donc que la troisième pour le nombre des maisons, dépassée par deux paroisses typiquement rurales.

Les paroisses du Gouvernement ont toutes leur église ou chapelle, sauf Gentilly: c'est le seul endroit, organisé comme paroisse, où l'on doive encore faire les offices religieux dans une maison privée. Six de ces paroisses n'ont pas de curé résidant et doivent être desservies par les curés du voisinage: Cap-de-la-Madeleine et Gentilly doivent compter sur les visites périodiques du curé de Champlain; Saint-Pierre-les-Becquets, sur celles du curé de Deschaillons; Maskinongé, sur celles du curé de Rivière-du-Loup, et Baie-Saint-Antoine 
sur celles du curé de Nicolet ${ }^{62}$. Dans la mission de Bécancour, les Abénaquis sont obligés de fréquenter l'église paroissiale, parce qu'ils n'ont plus d'église. Les douze curés résidants sont tous des prêtres séculiers, sauf ceux des Trois-Rivières et des Forges qui sont des récollets et celui de Bécancour qui est un jésuite; un autre jésuite fait fonction de missionnaire à Saint-François. Le grand-vicaire Perrault a donc sous sa direction neuf curés séculiers, deux curés récollets, un curé et un missionnaire jésuites; il peut en outre compter sur trois ou quatre récollets et jésuites pour les besoins du ministère.

Les écoles ne sont pas faciles à découvrir, peut-être parce qu'ici comme ailleurs, ce sont les curés qui se chargent, à titre privé, de montrer à lire et à écrire. Il semble bien que les Récollets dirigent aux Trois-Rivières une école pour garçons; et il s'y trouve peut-être aussi le maître d'école Denys Ponsant. Nous connaissons deux écoles pour filles: le pensionnat des Ursulines aux Trois-Rivières où quelquesunes des vingt religieuses se consacrent à l'éducation, et le pensionnat de Champlain tenu par deux Sœurs de la Congrégation.

Comme le Gouvernement possède son hôpital militaire (celui des Ursulines de la ville), il doit $\mathrm{y}$ avoir ici et là quelques médecins et chirurgiens: nous n'en retraçons que trois: Egon à Champlain, Alavoine aux Trois-Rivières et Rimbaud à Rivière-du-Loup.

Nous connaissons plus de notaires: sept, dont trois entrent en fonction immédiatement à l'inauguration du régime; Batiscan, Champlain, Maskinongé et Saint-François ont chacune le leur, TroisRivières en a trois $^{63}$.

Le développement agricole n'a rien de bien admirable; d'ailleurs, Burton dira bientôt que le Gouvernement ne produit pas assez de blé pour se suffire à lui-même ${ }^{64}$. Sur les 93,140 acres concédées, seulement 15,701, soit la sixième partie, sont mises en valeur: toute proportion gardée, c'est Saint-François qui est la plus avancée

62. Au moment où nous faisons la description du Gouvernement (automne de 1760), la Pointe-du-Lac a son curé résidant, mais elle le perd en décembre et sera ensuite desservie périodiquement par le récollet Pétrimoulx qui assiste son frère, curé de Rivière-du-Loup.

63. Augé, dont parle Roy (Histoire du notariat, II: 14), ne peut compter dans la liste de ce Gouvernement, puisque Deschaillons, ou il pratique, fait partie du Gouvernement de Québec.

64. Doc. const., 72. 
dans l'exploitation, Trois-Rivières vient en cinquième lieu, et Rivière-du-Loup arrive au dernier rang avec seulement la trente et unième partie de ses terres mises en valeur (nous ne comptons pas évidemment le village ouvrier des Forges). Le bétail se chiffre en tout à 1,123 chevaux, 3,106 bêtes à cornes et 894 moutons: SainteAnne est en tête pour l'élevage des chevaux et des moutons ${ }^{65}$, mais c'est a Yamaska qu'on trouve réuni le plus grand nombre de têtes de bétail: avec son chiffre de 650, elle laisse loin derrière elle les paroisses les plus riches ${ }^{66}$. La Pointe-du-Lac fait triste figure avec ses 20 chevaux et ses 50 bêtes à cornes; quant aux 10 moutons de Champlain, ils méritent d'être signalés à cause de leur rareté. "Il y a aussi dans ce gouvernement, ajoute le rapport, beaucoup de cochons, de volailles et d'oiseaux sauvages, surtout le pigeon des bois ${ }^{67}$. Le poisson abonde dans le lac Saint-Pierre, et aux environs de Noël, une abondance si étonnante de petits poissons ${ }^{68}$ remonte la rivière Saint-Maurice qu'elle suffit à nourrir un grand nombre de familles pauvres durant une grande partie de l'hiver. On s'en sert même pour nourrir les cochons"69.

C'est avec toutes ces ressources matérielles et humaines, avec l'industrie du fer qui reprend lentement, avec la fabrication des canots et le commerce de la fourrure, que le Gouvernement des TroisRivières entreprend une nouvelle période de son histoire, celle du régime militaire.

Marcel TRUdeL, professeur d'histoire du Canada a l'Universite Laval.

65. Ste-Anne: 130 chevaux et 208 moutons; Trois-Rivières, 80 chevaux et 50 moutons.

66. Ste-Anne, au deuxième rang, 590; Yamachiche, au troisième, 410.

67. Les fameuses tourtres, aujourd'hui disparues.

68. Les "petits poissons des Chenaux" ou "petites morues".

69. Doc. const., 76. 